\title{
Inteligencia emocional: influencia en la gestión de los recursos humanos en las organizaciones
}

\section{Emotional intelligence: influence on human resources management in organizations}

José María Biedma-Ferrer ${ }^{1}$

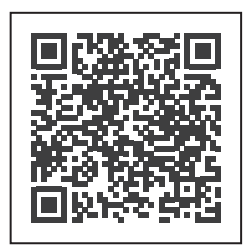

Articulo de revisión:

Fecha de recepción:

17/10/2020

Fecha de aceptación:

05/02/2021

Esta publicación se encuentra bajo licencia:

Creative Commons

Reconocimiento-

NoComercial-

SinObraDerivada 4.0

Internacional

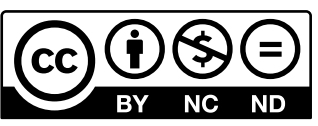

\section{Resumen}

La inteligencia emocional está adquiriendo una importancia creciente en las organizaciones, debido a las ventajas que se derivan de su utilización. El objetivo principal de este trabajo es analizar el impacto de este tipo de inteligencia sobre la selección de los empleados, la motivación, el rendimiento, el liderazgo y la toma de decisiones. La metodología empleada es la revisión sistemática de la literatura. Para ello, se han consultado 99 documentos en diversas bases de datos. Los resultados del trabajo constatan una relación positiva entre la inteligencia emocional y las variables analizadas. Asimismo, se propone un modelo que muestra el impacto de la mencionada inteligencia sobre las variables seleccionadas. Como futuras líneas de investigación, se insta a realizar más trabajos empíricos en el ámbito empresarial, que analicen, desde el punto de vista estadístico, las correlaciones señaladas por el modelo planteado.

Palabras claves: Inteligencia emocional, liderazgo, motivación rendimiento, selección de personal, toma de decisiones.

1 Licenciado en Ciencias Políticas, Licenciado en Derecho, Doctor en Derecho, Profesor del Departamento de Organización de Empresas de la Universidad de Cádiz, Instituto Universitario para el Desarrollo Social Sostenible (INDESS), España, josemaria.biedma@gm.uca.es https://orcid.org/0000-0002-5120-4447, Google Scholar 


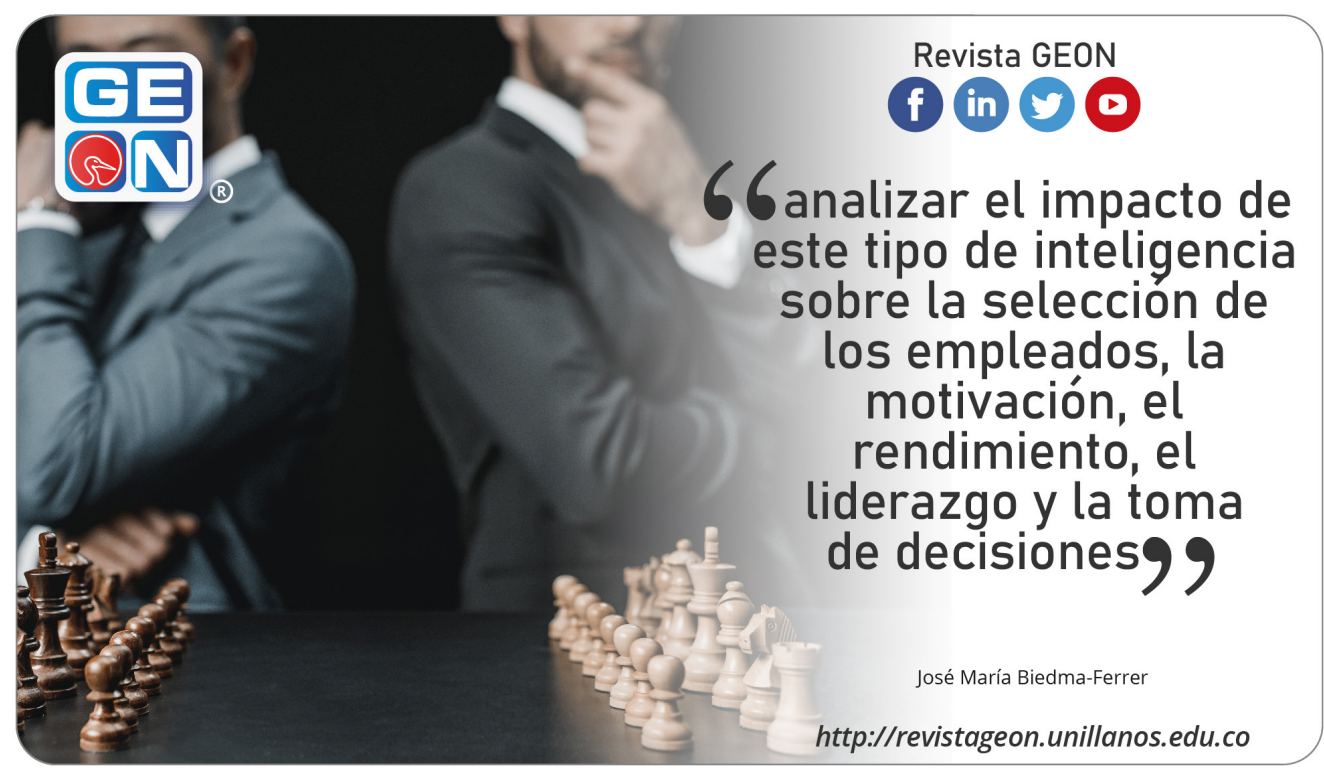

\section{Abstract}

Emotional intelligence is acquiring increasing importance in organizations, due to the advantages derived from its use. The main objective of this work is to analyze the impact of this type of intelligence on the selection of employees, motivation, performance, leadership and decision-making. The methodology used is based on a systematic review of the literature. For this, 99 documents have been consulted in various databases. The results of the work confirm a positive relationship between emotional intelligence and the variables analyzed. Likewise, an integrative model is proposed that shows the impact of the aforementioned intelligence on the selected variables. As future lines of research, it is proposed to carry out more empirical work in the business field that analyzes, from the statistical point of view, the correlations indicated by the proposed model.

Cómo citar este artículo / Toreference this article:

Biedma-Ferrer, J. M. (2021). Inteligencia emocional: influencia en la gestión de los recursos humanos en las organizaciones. Revista GEON (Gestión, Organizaciones Y Negocios), 8(1), 1-17. https://doi. org/10.22579/23463910.272

Keywords: Decision making; Emotional intelligence; Leadership; Motivation performance; Selection of personnel.

Códigos JEL: M54 Labor Management; M59 Personnel Economics: Other 


\section{Introducción}

Es indiscutible que la sociedad está experimentando un progreso tecnológico rápido y de gran importancia. También cabe señalar que las competencias sociopersonales están cobrando cada vez una mayor importancia en el ámbito profesional (Durán-Aponte y Durán-García, 2012). En este sentido, es básico que los empleados sean capaces de desarrollar aspectos relativos a la comunicación emocional (Pineda-Henao, 2020). Es decir, se trata de ser capaces de transmitir las propias emociones a otros sujetos, para lograr más efectividad en el mensaje (Gutiérrez y García, 2015). La comunicación emocional puede considerarse como la capacidad y habilidad que posee cada persona para la comprensión tanto de sus estados emocionales como los estados de los demás, y su transmisión por medio de una correcta expresión verbal y no verbal (Etchevers, 2008).

Las emociones, sin duda, son una parte de la esencia de las personas (Duque, García y Hurtado, 2017). Desde hace un tiempo, se viene estudiando el grado de influencia de las emociones en el comportamiento de los empleados en las organizaciones. En este sentido, se ha destacado la importancia del tema en el marco del análisis del comportamiento de las organizaciones (Feldman y Blanco, 2006; Ashkanasy, Hartel y Zerbe, 2000). En la literatura se ha evidenciado cierta correlación entre el adecuado ejercicio del líder y sus habilidades emocionales, debido a que estas son determinantes de sus compor- tamientos e interacciones (Subiran, 2010; Ljungholm, 2014). Asimismo, se ha puesto de manifiesto que los trabajadores trasladan sus emociones al sitio en el que desempeñan su trabajo (Robbins y Judge, 2017). Pero, además, como señalan López, Pulido y Augusto (2013), tratar de desvincular emociones y actividad laboral es una tarea muy difícil. Estos autores destacan la complementariedad de la racionalidad y las emociones, que cobran una especial importancia en la toma de decisiones, dado que los distintos estados afectivos en los que se hallen los decisores ejercen una gran influencia. El control de las emociones en las interacciones laborales es fundamental (Gracia y Martínez, 2004).

Dentro del contexto organizativo, la inteligencia emocional (IE) tiene cada vez un papel más importante. Se ha afirmado que, en el siglo XXI, la IE se va a convertir en la herramienta o recurso más importante para la supervivencia en un mundo en cambio permanente (Martín, 2013). Al respecto, en la literatura, se ha manifestado que la IE tiene aplicación en todos Ios seres humanos y en todas las etapas, así como en todas las profesiones y condiciones sociales (Cano y Zea, 2012). Cuando las personas se incorporan al mundo laboral, las empresas dan mucho valor a la competencia emocional de los sujetos, porque con frecuencia "marca la diferencia entre el éxito o el fracaso en la socialización y desarrollo de un individuo dentro de una organización" (Jiménez, 2018, p. 459). No obstante, cabe destacar que buena parte de la investigación en materia de emociones e IE se ha 
centrado en al área educativa (Trujillo y Rivas, 2005). Esto supone una brecha en el área de la investigación de organización de empresas. En esta línea, se ha señalado que los directivos suelen conocer poco aquello que sienten los demás (en referencia a los empleados) y presentan cierta dificultad para entablar amistad entre ellos mismos y los demás (Araujo y Leal, 2007).

Los objetivos del presente trabajo son: estudiar la creciente importancia de la IE en las organizaciones, revisar el concepto de IE en la literatura académica y analizar cómo la IE puede influir en aspectos tales como la selección de los empleados, el rendimiento, el liderazgo y la toma de decisiones.

El estudio se estructura del siguiente modo: en primer lugar, se realiza una introducción; en segundo lugar, se indican los objetivos y la metodología empleada; en tercer lugar, se revisan las principales definiciones de la IE en la literatura académica, abordando los principales argumentos a favor y en contra de la IE; en cuarto lugar, se profundiza en la influencia e impacto de la IE en diversas variables relacionadas con las organizaciones y los recursos humanos. Finalmente, se indican las conclusiones.

\section{Marco teórico}

Para cualquier persona que forme parte del equipo directivo de una organización resulta de interés conocer la definición de IE, con el fin de que pueda aplicarla en sus relaciones con los empleados. Por ello, es importan- te revisar el concepto de IE y su evolución a lo largo del tiempo. Aunque existe poca información sobre este tema, una investigación empírica llevada a cabo por Ponti y Cabañas (2004) consideró que más de la mitad de los directivos españoles encuestados no conocía la definición de IE. Por otra parte, un estudio realizado en Colombia señaló que los líderes del sector financiero tienen dificultades para la percepción y el entendimiento de las emociones de las personas que tienen a su alrededor (Zárate y Matviuk, 2010).

\section{Concepto de inteligencia emocional}

La IE ha sido definida en la literatura de muy diversa forma y no existe un consenso sobre el concepto (Brackett y Geher, 2006; Sternberg, Castejón, Prieto, Hautamäki y Grigorenko, 2001). En este apartado, se muestran diversas formas de entender la $\mathrm{IE}$, establecidas a lo largo del tiempo, con el fin de tratar de esclarecer el concepto.

En una visión retrospectiva, fue Thorndike (1920) quien hizo referencia al concepto de inteligencia social. Como lo ha señalado Law et al. (2008), Thorndike definió la inteligencia social como aquella capacidad de entendimiento y manejo de las personas, con el fin de proceder de forma sabia en las relaciones humanas. Desde el punto de vista formal, la primera definición de IE fue publicada en 1990 por Mayer y Salovey. Más adelante, y tras considerarse a la IE como una inteligencia más, la IE se definió como 
una colección de habilidades alcanzadas por las personas, con fines de tipo adaptativo (Mayer et al., 1999). El concepto de IE logró gran popularidad gracias al psicólogo Goleman, con la publicación de su libro Inteligencia Emocional en 1985.
En la tabla 1 se muestran, por orden cronológico, las diversas definiciones de la IE extraídas de la revisión de la literatura, que pueden servir de ayuda para una mejor comprensión del concepto por parte de profesionales y de académicos.

Tabla 1. Revisión del concepto de IE en la literatura

\begin{tabular}{|c|c|}
\hline Autor & Aportación al concepto de inteligencia emocional \\
\hline Goleman (1995) & $\begin{array}{l}\text { Capacidad que tienen las personas para la gestión de las emociones y la utili- } \\
\text { zación en favor de un mayor desempeño laboral. }\end{array}$ \\
\hline Cooper y Sawaf (1997) & $\begin{array}{l}\text { Aptitud para conseguir captar, entender y aplicar de forma eficaz tanto la } \\
\text { fuerza como la perspicacia de las emociones, dado que son fuente de la ener- } \\
\text { gía humana, la información y las relaciones e influencia. }\end{array}$ \\
\hline Mayer y Salovey(1997) & $\begin{array}{l}\text { Capacidad para manejar los sentimientos y las emociones propias y de las } \\
\text { demás personas, discriminando entre ellos y aprovechando los conocimien- } \\
\text { tos para dirigir los propios pensamientos y acciones. }\end{array}$ \\
\hline Ryback (1998, p. 325) & $\begin{array}{l}\text { "La capacidad de aplicar la conciencia y la sensibilidad para discernir los senti- } \\
\text { mientos y subyacentes de la comunicación interpersonal". }\end{array}$ \\
\hline Goleman (1998) & $\begin{array}{l}\text { Capacidad para el reconocimiento tanto de los sentimientos propios como de } \\
\text { los ajenos, la motivación y el manejo de forma adecuada de las relaciones que } \\
\text { mantenemos nosotros mismos y con los demás. }\end{array}$ \\
\hline Bar-On (2000) & $\begin{array}{l}\text { Es un agregado de habilidades y conocimientos relativos a lo emocional y } \\
\text { social, que influye en la capacidad general para poder afrontar de modo efec- } \\
\text { tivo las demandas de nuestro medio. }\end{array}$ \\
\hline Bradberry (2009) & $\begin{array}{l}\text { Es aquello que las personas llevan dentro, de carácter intangible, que tiene } \\
\text { influencia en cómo manejamos el comportamiento, en la forma de atravesar } \\
\text { las complejidades sociales y en cómo llevamos a cabo la toma de decisiones } \\
\text { personales que posibilitan lograr resultados positivos. }\end{array}$ \\
\hline $\begin{array}{l}\text { Fernández y Ramos } \\
\text { (2002) }\end{array}$ & $\begin{array}{l}\text { Supone la capacidad para poder reconocer, comprender y regular tanto } \\
\text { nuestras emociones como las ajenas. }\end{array}$ \\
\hline Cooper y Sawaf (2004) & $\begin{array}{l}\text { Es la capacidad de sentir, entender y aplicar eficazmente el poder y la agu- } \\
\text { deza de las emociones, estimadas como origen de la información, la energía } \\
\text { humana, la conexión y la influencia. }\end{array}$ \\
\hline Chopra y Kanji (2010) & $\begin{array}{l}\text { Es aquella que contribuye a la generación de cualidades de líder, a la activación } \\
\text { de conocimientos y a dirigir a las personas de forma efectiva, entre otros aspec- } \\
\text { tos. }\end{array}$ \\
\hline $\begin{array}{l}\text { García-Fernández y } \\
\text { Giménez-Más (2010) }\end{array}$ & $\begin{array}{l}\text { Es la capacidad del ser humano para adaptarse e interaccionar con el } \\
\text { entorno, dinámico y cambiante, a partir de sus propias emociones. }\end{array}$ \\
\hline $\begin{array}{l}\text { Zardeni y Yousefi } \\
(2012)\end{array}$ & $\begin{array}{l}\text { Es aquel tipo de inteligencia que permite a las personas examinar sus emocio- } \\
\text { nes, así como la conducción de sus pensamientos y actitudes, posibilitando ree- } \\
\text { laborar y corregir las percepciones, así como la evaluación de las emociones. }\end{array}$ \\
\hline Alkozei et al. (2015) & Capacidad de entender, percibir y gestionar las emociones. \\
\hline Robbins y Judge (2017) & Aptitud para detectar y manejar tanto señales como información emocional. \\
\hline
\end{tabular}

Fuente: elaboración propia a partir de Goleman (1995); Cooper y Sawaf (2004); Mayer y Salovey (1997); Ryback (1998, p. 325); Goleman (1998); Bar-On (2000); Bradberry (2009); Fernández y Ramos (2002); Cooper y Sawaf (2004); Chopra y Kanji (2010); García-Fernández y Giménez-Más (2010); Zardeni y Yousefi (2012); Alkozei et al. (2015); y Robbins y Judge (2017). 
Tras la revisión de la literatura sobre el concepto de IE, se evidencia la existencia de múltiples definiciones de este tipo de inteligencia como consecuencia de la inexistencia de consenso en la literatura (Brackett y Geher, 2006; Sternberg et al., 2001). No obstante, es preciso avanzar en este debate y proponer definiciones integradoras. En este sentido, se realiza la siguiente propuesta: la IE se puede definir como la capacidad de conocer y manejar tanto las emociones propias como las de terceras personas, que permite la reelaboración y la corrección de las percepciones y la evaluación de las emociones. A través de la $\mathrm{IE}$, se podrán obtener conocimientos suficientes, con el fin de mejorar tanto la dirección de los pensamientos y acciones propias como las de los demás.

La existencia de una pluralidad de definiciones también conlleva el estu- dio de las ventajas y desventajas de la utilización de la IE, que se estudian a continuación.

\section{Ventajas y desventajas de la utilización de la inteligencia emocional}

Como ya se indicó, la falta de consenso sobre el concepto de IE se refleja en las argumentaciones esgrimidas a favor y en contra de la IE. En efecto, en la revisión de la literatura, se observó la existencia de cierto debate sobre las ventajas y desventajas de la utilización de la IE en las organizaciones.

En la tabla 2 se indican las principales ventajas de la utilización de la IE.

De la tabla 2 se desprende que existen argumentos de peso para que los directivos apliquen la IE en sus organizaciones, de acuerdo con los estudios realizados.

Tabla 2. Principales ventajas de la utilización de la IE

\begin{tabular}{ll}
\multicolumn{1}{c}{ Autor } & \multicolumn{1}{c}{ Argumento } \\
Jiménez (2018) & La competencia emocional es altamente valorada por las empresas. \\
\hline Solano (2013) & $\begin{array}{l}\text { La IE puede contribuir a favorecer el crecimiento profesional de los emplea- } \\
\text { dos. }\end{array}$ \\
\hline Robbins y Judge (2017) & $\begin{array}{l}\text { Las personas que pueden percibir las emociones ajenas son capaces de } \\
\text { controlar las suyas propias y logran controlar las interacciones sociales. }\end{array}$ \\
\hline Robbins y Judge (2017) & $\begin{array}{l}\text { La IE puede ser un buen predictor de criterios relevantes. Por ejemplo, la IE } \\
\text { puede ser un buen predictor del desempeño laboral y de la evaluación de } \\
\text { los empleados. }\end{array}$ \\
\hline Danvila y Sastre (2010) & $\begin{array}{l}\text { La IE presenta utilidad. En el sector de la consultoría, se considera una } \\
\text { herramienta de gran aceptación. }\end{array}$ \\
\hline Bar-On et al. (2003) & $\begin{array}{l}\text { La IE presenta un fundamento neurológico que no guarda relación con las } \\
\text { medidas de carácter estándar que tiene la inteligencia. } \\
\text { Cherniss y Adler (2000) }\end{array}$ \\
\hline $\begin{array}{l}\text { La IE proporciona las bases de las competencias necesarias e importantes } \\
\text { para mayoría de los trabajos. }\end{array}$ \\
\hline
\end{tabular}

Fuente: elaboración propia a partir de Jiménez (2018); Solano (2013); Robbins y Judge (2013); Danvila y Sastre (2010); Bar-On et al. (2003); y Cherniss y Adler (2000). 
En la tabla 3 se muestran las principales desventajas de la utilización de la IE.

Tabla 3. Principales desventajas de la utilización de la IE

\begin{tabular}{l|l}
\multicolumn{1}{c|}{ Autor } & \multicolumn{1}{c}{ Argumento } \\
$\begin{array}{l}\text { Brackett y Geher } \\
\text { (2006) }\end{array}$ & $\begin{array}{l}\text { Falta de consenso con } \\
\text { respecto al concepto } \\
\text { de IE. }\end{array}$ \\
\hline $\begin{array}{l}\text { Matthews, } \\
\text { Zeidner y Roberts } \\
\text { (2002) }\end{array}$ & $\begin{array}{l}\text { La IE es más un mito que } \\
\text { una ciencia. }\end{array}$ \\
\hline & $\begin{array}{l}\text { No existe consenso en la } \\
\text { literatura para definir el } \\
\text { constructo IE. }\end{array}$ \\
& $\begin{array}{l}\text { Diversidad y escaso rigor } \\
\text { en la forma de medir la IE. }\end{array}$ \\
\hline
\end{tabular}

Fuente: elaboración propia a partir de Brackett y Geher (2006); Matthews, Zeidner y Roberts, (2002); Locke (2005); y Conte (2005).

Tras la exposición de las ventajas y desventajas de la utilización de la IE a través de la literatura, se concluye que los diversos trabajos existentes muestran cierta controversia al respecto. No obstante, cabe afirmar que los beneficios de la utilización de la IE tienen mayor peso que las desventajas.

\section{Metodología}

Desde un punto de vista metodológico, el presente trabajo se ubica en los procesos de revisión documental del estado del arte, señalados por Carramolino (2009). Como señala esta autora, el estado de conocimiento constituye una parte muy importante en el proceso de investigación. Se realizó una revisión sistemática de la literatura como técnica exploratoria y analítica para la recolección de información, análisis y tratamiento de aquella que presenta interés para los objetivos del trabajo. Dicha revisión ha comprendido artículos científicos en revistas indexadas, comunicaciones publicadas y monografías de editoriales de prestigio internacional. Para el presente trabajo, se revisaron 99 documentos. Las principales bases de datos utilizadas fueron Web of Knowledge, ABI INFORM, Latindex y Dialnet.

Con el fin de obtener publicaciones de interés, se filtró la búsqueda de aquellos documentos cuyas palabras clave -tanto en inglés como en español- guardaran relación con los objetivos de la investigación, tales como: inteligencia emocional, emoción, selección de personal, rendimiento, liderazgo, toma de decisiones. Después de esto, se procedió al tratamiento de la información para la elaboración del presente trabajo. La redacción del trabajo se realizó a partir de los hallazgos consolidados o ideas más relevantes de las investigaciones analizadas.

\section{Resultados}

Para los directivos, tanto la gestión adecuada de sus propias emociones como de los empleados tiene consecuencias en determinados aspectos, que se tratan a continuación. En la literatura, se ha considerado que la IE de los directivos puede tener cierta influencia en la capacidad de aprendizaje organizativo de los empleados (Suárez y Trespalacios, 2011). Por otra parte, algunas investigaciones han mostrado que la IE tiene una incidencia considerable en el ámbito laboral (Prati et al., 2003; Voola, Carlson y West, 2004; Dulewicz y Higgs, 2004). 
A los directivos, y a las organizaciones, en general, les resulta de utilidad el conocimiento de la influencia que puede tener la inteligencia emocional en la gestión de los recursos humanos (Torres-Flórez, 2019). Así, por ejemplo, la IE en los directivos puede ayudar a las organizaciones a reducir el abandono laboral, reteniendo empleados valiosos (Mohammad et al., 2014; Avey, Luthans y Jensen, 2009). A partir de estas consideraciones, se plantean los siguientes objetivos de investigación: el estudio de la importancia de la IE en los procesos de selección de personal; la posible correlación entre IE motivación; la posible correlación entre IE y el rendimiento; los efectos de la IE sobre el liderazgo; y, finalmente, comprobar en qué medida la IE puede ser un instrumento para la mejora del proceso de toma de decisiones de los directivos. La elección de estas cinco variables se justifica por la importancia que tienen en la gestión de los recursos humanos, según se ha señalado en diferentes trabajos (Domínguez, 2008; Robbins y Judge, 2017).

\section{Inteligencia emocional y selección de personal}

Sassha y Tumaev (2007) han afirmado acertadamente que una de las preocupaciones más importantes para los directores de recursos humanos, jefes de departamento o encargados de la selección de personal es encontrar la persona más adecuada y competente para el puesto que se desea contratar.

Debido a la importancia de la selección y la dificultad de realizar una bue- na selección, para las organizaciones es interesante saber hasta qué punto el empleo de la IE en los procesos de selección es beneficioso para ellas. Se ha considerado que en la selección de personal es importante contar con la IE (Atalaya, 2001). En la misma línea, Toledo y Salvador (2014) han señalado que el conocimiento del nivel de competencia emocional de las personas en el proceso de selección es beneficioso.

En los procesos de selección es necesario contratar a personas con competencias en IE, además de las competencias técnicas. Por ello, las empresas actuales demandan características personales que se encuentren estrechamente relacionadas con la IE (Bresó y Megías, 2012; Seco, 2006). En este sentido, se ha investigado que los empleados emocionalmente inteligentes se adaptan mejor y de forma más rápida a los nuevos puestos (Seco, 2006). También existen estudios que han demostrado que la ausencia de competencias y habilidades emocionales provoca el despido de los trabajadores (Bresó y Salanova, 2010). Cada vez son más los empleadores que usan medidas de IE a la hora de contratar personal (Robbins y Judge, 2017), porque consideran que la IE puede servir como criterio en las organizaciones para la selección de personal (Caballero y Blanco, 2007). En la misma línea, Seco (2006) ha advertido que los departamentos de personal están aplicando la IE en los procesos de selección de personal. En el caso de la selección de ejecutivos de alta dirección, se ha constatado la relevancia de la IE en el éxito de la alta dirección (Fernández-Aráoz, 2005). 
A partir de las consideraciones anteriores, se puede formular la siguiente proposición:

Proposición n. ${ }^{0}$ 1. La utilización de la IE en los procesos de selección de personal puede tener importantes beneficios para las organizaciones.

\section{La inteligencia emocional y la motivación}

La motivación, sin duda, tiene un papel muy significativo en la explicación del comportamiento humano (Herrera et al., 2004). En este sentido, cabe señalar que la motivación ha adquirido notable importancia en múltiples ámbitos de la vida, entre los que se encuentra la educación.

De acuerdo con Ajello (2003), se puede señalar que la motivación debe ser entendida como la trama que se apoya en el desarrollo de las actividades consideradas importantes para el sujeto y en las que toma parte. Desde el punto de vista educativo, la motivación puede entenderse como la disposición en sentido positivo para el aprendizaje continuo de forma autónoma.

Se ha estudiado que el acierto en la forma de motivar conlleva que los trabajadores tengan mayor sentido de pertenencia, con mejores resultados en las actividades que realicen. Todo ello contribuirá al éxito de la organización a la que pertenecen los empleados (Peña y Villón, 2018). Anteriormente, se investigó que cuando el trabajador tiene sentimientos de éxito en la ejecución de una tarea, aumenta la motivación y la satisfacción en el trabajo (Cherniss, 1993). Esta investigación también concluyó que cuando no se logra el éxito, el individuo tiende a desconectarse y muestra poco interés. Ilies y Judge (2005) señalan que la retroalimentación a los empleados sobre su desempeño influye en su estado de ánimo, lo que supone una mejora del desempeño. Con posterioridad, Tsai, Chen y Liu (2007) consideraron que, si se parte de que la motivación puede suponer una mejora del desempeño del empleado, la productividad se incrementa en estos casos y se obtienen mejores reportes de los supervisores sobre el desempeño logrado. Recientemente, se ha considerado que la falta de motivación en los empleados afecta al rendimiento laboral, con implicaciones para la salud emocional y psicológica (Gazca, Zárate y Mota, 2020).

En el ámbito educativo, también se ha demostrado la existencia de cierta relación entre la motivación y la inteligencia emocional percibida, entendiendo esta última "como un conjunto de habilidades de procesamiento emocional" (Arias y Giuliani, 2014, p. 123). No obstante, son necesarias más investigaciones para corroborar dicha relación (Cera, Alamgro, Conde y Saenz-López, 2015).

Proposición n. ${ }^{\circ}$ 2. La utilización de la IE puede influir en la motivación del sujeto.

\section{La inteligencia emocional y el liderazgo}

En los estudios de investigación se ha considerado que todo trabajo 
es emocional y que las emociones positivas son la base del liderazgo (López et al., 2013). Cabe señalar que las emociones positivas son reacciones breves experimentadas cuando se produce algo significativo para el sujeto (Contreras y Esguerra, 2006). También se ha entendido que el lado emocional de la dirección de las organizaciones proporciona vida y significado a las estructuras directivas (Barach y Eckhardt, 1996, citado en Caruso y Salovey, 2005). En este sentido, Ashkanasy y Daus (2002) han considerado una relación positiva entre el éxito de los líderes capaces del reconocimiento a sus empleados y del manejo tanto de sus propias emociones como la de los demás. Además, también se ha estudiado que los trabajadores que logran mayores puntuaciones en IE tienen un gran potencial de liderazgo (Lopes, Cote, y Salovey, 2006). En esta línea, una investigación posterior evidenció una correlación positiva entre las variables IE y liderazgo femenino en los puestos directivos en organismos públicos (Batista y Bermudez, 2009). Por tanto, aquellos líderes que conducen las emociones de forma negativa imposibilitan que sus empleados puedan alcanzar el éxito (Bradberry y Greaves, 2009). Por otra parte, se ha considerado que los líderes amantes de innovar y los directivos eficaces se caracterizan por el uso de la IE (Cadman y Breuer, 2001).

De acuerdo con la literatura revisada, se puede afirmar que la IE contribuye a la efectividad de los líderes, porque las emociones tienen un papel clave en el proceso de liderazgo (George, 2000). De hecho, se ha considerado que los líderes eficaces utilizan los recursos emocionales para la transmisión de sus mensajes (Lewis, 2000). Los líderes deben realizar un buen manejo de las emociones. Por tanto, aquellos que conducen sus emociones de forma positiva pueden lograr lo mejor de aquellos que le siguen. Por el contrario, los líderes que manejan sus emociones de forma negativa dificultan o imposibilitan a sus seguidores para alcanzar el éxito en el desempeño de su trabajo (Bradberry y Greaves, 2012). También se ha puesto de manifiesto la relación de dependencia entre el pensamiento y los sentimientos. Esto implica que aquellas personas que utilizan de forma correcta las emociones contribuyen al fomento del pensamiento, habilitándoles para motivar, inspirar o ilusionar a los demás sujetos, siendo esto la esencia del liderazgo (Caruso y Salovey, 2005).

Finalmente, cabe señalar que los directivos de las grandes empresas tienen conciencia de la importancia de evidenciar entusiasmo emocional ante sus empleados, para que ellos acepten con mayor facilidad su visión sobre la organización y la actitud ante el cambio (Robbins y Judge, 2010). En este sentido, se ha señalado que aquellas personas que autogestionan bien sus emociones, percibiendo y comprendiendo las emociones de los demás, son capaces de desarrollar competencias laborales tales como el liderazgo (Duque et al., 2017). Además, se ha considerado que las competencias emocionales transversales de tipo comunicativo, tales como el liderazgo, tienen una alta relación con la empleabilidad (Briz, 2016). 
Con base en lo anteriormente expuesto, se puede plantear la siguiente proposición:

Proposición n. ${ }^{0}$ 3. La utilización de la IE por parte de los líderes tiene efectos positivos para las organizaciones.

\section{La inteligencia emocional y el rendimiento}

La relación positiva entre la IE y el rendimiento en las organizaciones ha sido señalada en la literatura por la existencia de una gran correspondencia entre el rendimiento y habilidades tales como la conducción de las emociones o el manejo de la frustración (Cartwright y Pappas, 2008). En este sentido, se ha evidenciado que los sujetos con mayor nivel de IE influyen en la mejora de resultados de las organizaciones (Dulewicz y Higgs, 2004; Voola et al., 2004). También se ha puesto de manifiesto que cuanto mayor complejidad tiene el trabajo, más necesaria es la inteligencia emocional (Araujo y Leal, 2007). Al respecto, se ha señalado que los altos directivos difieren en rendimiento principalmente por su IE (Watkin, 2002; Langhorn, 2004). Más recientemente, se ha encontrado una relación positiva entre IE y desempeño, pero sin hacer referencia al tipo de trabajo de que se trata (Carmeli y Josman, 2006; Rangarajan y Jayamala, 2014). Por otra parte, otras investigaciones han confirmado también dicha relación, pero señalando que esta no es fuerte (Rooy y Viswesvaran 2004; O’Boyle et al., 2011). Sería conveniente realizar más estudios empíricos sobre este tema.
También resulta de interés estudiar la posible relación entre la IE y el rendimiento de los equipos de trabajo. Se ha establecido una correlación positiva entre los equipos de trabajo que poseen unos niveles de IE elevados y la mejora del rendimiento, porque se adaptan más fácilmente a las diferentes formas de realizar las tareas (López et al., 2013). Además, aquellos equipos de trabajo que poseen una elevada IE, al inicio de la actividad, cuentan con una capacidad de ponerse en marcha mucho más rápidamente que los equipos con IE baja (Jordan et al., 2002).

Los argumentos indicados permiten realizar la siguiente proposición:

Proposición n. 4. La utilización de la IE puede ayudar a mejorar el rendimiento de los empleados en las organizaciones.

\section{La inteligencia emocional y la toma de decisiones}

La toma de decisiones en las empresas constituye una cuestión de importancia, dado que puede afectar al hecho de que una organización sea más o menos eficiente o competitiva, lo que puede llegar al extremo del cierre de esta, debido a la toma de malas decisiones (Pérez, Cruz, Guatemala y Juárez, 2018).

Se ha estudiado que razón y emoción se unen en la vida diaria de forma constante e inconsciente. Por ello, una buena parte de la información emocional se procesa de forma inconsciente y esto afecta a nuestro 
modo de pensar (Jiménez-Ortega et al., 2017). Si las emociones tienen importancia para el ejercicio del uso de la razón y marcan el rumbo de las decisiones de forma constante (Murcia y Zea, 2012), también cabe destacar que la toma de decisiones se encuadra dentro de un proceso complejo, en el que intervienen factores de índole personal e interpersonal (Bisquerra y Pérez, 2007).

Partiendo de lo indicado anteriormente, las organizaciones deben tener presente que las emociones y los estados de ánimo influyen de forma considerable en la toma de decisiones (Robbins y Judge, 2017). La investigación de la IE como herramienta gerencial para la mejora del proceso de toma de decisiones de directores y jefes ha señalado que existe una correlación alta y positiva entre la variable IE y la variable toma de decisiones (Labarca y Estaba, 2010). Recientemente, se ha entendido que las emociones desempeñan un papel muy importante dentro del proceso cognitivo de la toma de decisiones, porque actúan como una brújula que dirige al decisor hacia el camino correcto (Loria, 2018).

Por otra parte, Damasio (2010) ha estudiado la relación entre las emociones y la toma decisiones señalando que las emociones ayudan a tomar decisiones apropiadas, debido a que relacionan acciones o resultados que resultan positivos o negativos. En esta línea, un estudio realizado por Ramírez y Zavaleta (2018, p. 77) en una empresa concluyó que "a mayor inteligencia emocional, el trabajador tomará mejores decisiones".

Lo anteriormente expuesto permite establecer la siguiente proposición:

Proposición n. ${ }^{\circ}$ 5. La IE es una herramienta que ayuda en la toma de decisiones.

En la figura 1 se indican las posibles relaciones de la IE con las variables estudiadas, a partir de las proposiciones establecidas. Como puede observarse, se establece un vínculo positivo entre la IE y la selección de personal, la motivación, el liderazgo, el rendimiento de los empleados y la toma de decisiones.

Figura 1. Impacto de la IE sobre las distintas variables estudiadas.

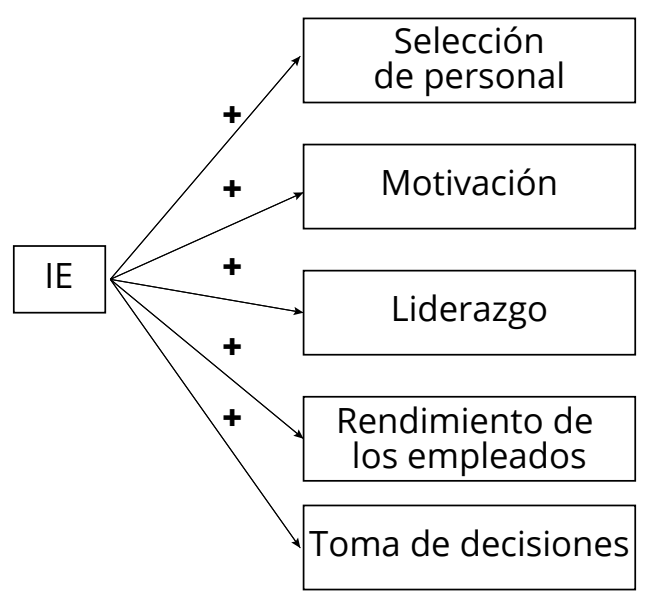

Fuente: elaboración propia a partir de Atalaya (2001); Toledo y Salvador (2014); Robbins y Judge (2017); Ilies y Judge (2005); Tsai et al. (2007); Cera, Conde Saenz-López (2015); López et al. (2013); Ashkanasy y Daus (2002); Watkin (2002); Langhorn (2004); Carmeli y Josman (2006); Cartwright y Pappas (2008); Rangarajan y Jayamala (2014); Damasio (2010); y Labarca y Estaba (2010). 


\section{Conclusiones}

Buena parte de la investigación en materia de emociones e IE se ha centrado principalmente en el área educativa. Esto supone una brecha en el área de la investigación de organización de empresas. Los directivos suelen conocer poco sobre aquello que los empleados sienten (Araujo y Leal, 2007) y desconocen cuál es la definición de IE. Se constata la existencia de múltiples y diversas definiciones de IE debido a la falta de consenso en la literatura. Ante esta situación, se propone una definición integradora que puede servir tanto para los profesionales como para los investigadores. La IE se puede definir como la capacidad de conocer y manejar tanto las emociones propias como las de los demás, lo que posibilita la reelaboración y la corrección de las percepciones y la evaluación de las emociones. A través de la IE se podrán obtener conocimientos suficientes, con el fin de mejorar tanto la dirección de los pensamientos y acciones propias como las de los demás.

Tras el estudio en la literatura de las ventajas e inconvenientes de la IE, se confirma que la utilización de esta tiene más ventajas que desventajas. En este sentido, cabe destacar que dicha inteligencia proporciona las bases de competencias necesarias e importantes para la mayoría de los trabajos (Cherniss y Adler, 2000).

Este trabajo propone un modelo que muestra las relaciones positivas entre la IE y cinco variables importantes en la gestión de los recursos humanos: la selección de personal, la motivación, el liderazgo, el rendimiento y la toma de decisiones. Ello supone una aportación interesante para la literatura, porque dichas relaciones no han sido estudiadas hasta el momento de forma conjunta. También resulta de gran interés para los profesionales que se encargan de dirigir las organizaciones.

El trabajo tiene como principal limitación ser un estudio de corte teórico, dado que se realiza una revisión de la literatura sobre los distintos temas tratados. No obstante, aporta a la literatura científica un punto de vista integrador del tópico analizado que ha sido estudiado en otras investigaciones de forma fragmentada y propone reflexionar sobre la relación entre la IE y las variables estudiadas, con el fin de servir de utilidad para los directivos de las organizaciones. Finalmente, como futuras líneas de investigación, se propone realizar más trabajos empíricos en el área de organización de empresas, que estudien, desde un punto de vista estadístico, la relación entre la IE y las distintas variables estudiadas, con el fin de corroborar que se cumplen las proposiciones indicadas en el trabajo. También sería interesante analizar las citadas relaciones en función de distintos sectores laborales, de la edad, del sexo y de otras variables sociodemográficas.

\section{Referencias}

Ajello, A. M. (2003). La motivación para aprender. En Manual de psicología de la educación pp. 251-271 Popular.

Alkozei, A., Schwab, Z. J. \& Killgore, W. D. (2015). The Role of Emotional Intelli- 
gence During an Emotionally Difficult Decision-Making Task. Journal of Nonverbal Behavior, 40(1), 39-54. https:// bit.ly/3d7yH3X

Araujo, M. \& Leal, M. (2007). Inteligencia emocional y desempeño laboral en las instituciones de Educación Superior Públicas. Centro de Investigación de Ciencias Administrativas y Gerenciales, $4(2), 132-147$.

Arias, C. J. \& Giuliani, M. F. (2014). Explorando la Inteligencia Emocional Percibida en tres áreas etarias: un estudio realizado en Argentina. Estudos Interdisciplinares Sobre o Envelhecimento, 19(1),123-140. https://bit.ly/3tSsqit

Atalaya, M. (2001). Nuevos enfoques en selección de personal. Revista de Investigación en Psicología, 4(2), 133-144. https://bit.ly/3d7502W

Ashkanasy, N. M. \& Daus, C. S. (2002). Emotion in the workplace: The new challenge for managers. Academy of Management Perspectives, 16(1), 76-86. https://doi.org/10.5465/ ame.2002.6640191

Ashkanasy, N. M., Härtel, C. E. \& Zerbe, W. J. (2000). Emotions in the Workplace: Research, Theory, and Practice. Quorum Books.

Avey, J. M., Luthans, F. \& Jensen, S. M. (2009). Psychological capital: A positive resource for combating employee stress and turnover. Humain Resource Management, 48, 677-693. https://doi. org/10.1002/hrm.20294

Barach, J. A. \& Eckhardt, D. R. (1996). Leadership and the Job of the Executive. Quorum Books.

Bar-On, R. (2000). Emotional and social intelligence: insights from the emotional quotient inventory (EQ-I). En The
Handbook of Emotional Intelligence (pp. 363-388). Jossey-Bass.

Bar-On, R., Tranel, D., Denburg, N. L. \& Bechara, A. (2003). Exploring the neurological substrate of emotional and social intelligence. Brain, 126, 17901800.

Batista, J. \& Bermúdez, M. C. (2009). Inteligencia emocional y liderazgo femenino en cargos de dirección en organismos público. Revista Electrónica de Humanidades, Comunicación y Educación Social, 7(4), 45-65. https://bit. ly/3aXSpN3

Bisquerra, R. \& Pérez, N. (2007). Las competencias emocionales. Educación, 21(10), 61-82. https://doi. org/10.5944/educxx1.1.10.297

Brackett, M. A. \& Geher, G. (2006). Measuring emotional intelligence: Paradigmatic diversity and common ground. En Emotional intelligence and everyday life (pp. 27-50). Psychology Press.

Bradberry, T. \& Greaves, J. (2012). Leadership 2.0. Talentsmart.

Bradberry, J. \& Greaves, J. (2009). Emotional intelligence 2.0. TalentSmart.

Bresó, E. \& Megías, M. J. (2012). La razón y la emoción e la prevención de riesgos laborales: importancia de la inteligencia emocional en la selección y formación. Gestión Práctica de Riesgos Laborales, (94), 40-45. https://bit.ly/3jGtOAg

Bresó, E. \& Salanova, M. (2010). Organizaciones emocionalmente inteligentes como antídoto a los riesgos psicosociales. Gestión Práctica de Riesgos Laborales, (67), 12-16. https://bit.ly/2Nn8bJe

Briz, E. (2016). Inteligencia emocional y competencia de comunicación oral desde la perspectiva de la empleabilidad. En Inteligencia emocional y bien- 
estar: reflexiones, experiencias profesionales e investigaciones (pp. 419-437). Ediciones Universidad San Jorge.

Bolton, S. C. (2000). Emotion here, emotion there, emotional organizations every where. Critical Perspectives on Accounting, 11, 155-171. https://doi. org/10.1006/cpac.1998.0236

Caballero, D. \& Blanco, A. (2007). Competencias para la flexibilidad: la gestión emocional de las organizaciones. Psicothema, 19(4), 616-620. http://www. psicothema.com/pdf/3406.pdf

Cadman, C. \& Brewer, J. (2001). Emotional intelligence: a vital prerequisite for recruitment in nursing. Journal of Nursing Management, 9(6), 321-324. https://bit. Iy/2NjKDF8

Cano, S. \& Zea, M. (2012). Manejar las emociones, factor importante en el mejoramiento de la calidad de vida. Revista Logos Ciencia \& Tecnología, 4(1), 58-67. https://bit.ly/3tQ4exk

Carmeli, A. \& Josman, Z. E. (2006). The Relationship Among Emotional Intelligence, Task Performance, and Organizational Citizenship Behaviors. Human Performance, 19(4), 403-419. https:// bit.ly/3rUkeg9

Carramolino, B. (2009). La revisión del estado del arte. Metodología de la investigación. Universidad de Valladolid.

Caruso, D. R. \& Salovey, P. (2005). El directivo emocionalmente inteligente: $L a$ Inteligencia Emocional en la Empresa. EDAF.

Cartwright, S. \& Pappas, C. (2008). Emotional intelligence, its measurement and implications for the workplace. International Journal of Management Reviews, 10(2), 149-171. https://bit. $|y / 3 d 4 \times 5| \mid$
Cera, E., Almagro, B. J., Conde, C. \& SaenzLópez, P. (2015). Inteligencia emocional y motivación en educación física en secundaria. Retos, 27, 8-13. https:// bit.ly/3rRRzrl

Cherniss, C. (1993). The role of professinal self-efficacy in the etiology of burnout. En Professional burnout: Recent developments in theory and research (pp. 135-149). Taylor \& Francis.

Cherniss, C. \& Adler, M. (2000). Promoting emotional intelligence in organizations. ASTD.

Chopra, P. \& Kanji, G. (2010). Emotional intelligence: a catalyst for inspirational leadership and management excellence. Total Quality Management \& Business Excellence, 21(10), 971-1004. https://bit.ly/3qlpk4w

Conte, J. M. (2005). A review and critique of emotional intelligence measures. Journal of Organizational Behavior, 26(4), 433-440. $\quad$ https://doi.org/10.1002/ job.319

Contreras, F. \& Esguerra, G. (2006). Psicología positiva: una nueva perspectiva en psicología. Diversitas, 2, 311-319. https://bit.ly/2OAxYOD

Cooper, R. \& Sawaf, A. (1997). Estrategia emocional para ejecutivos. Martínez Roca.

Cooper, R. \& Sawaf, A . (2004). La inteligencia emocional aplicada al liderazgo y a las organizaciones. Grupo Editorial Norma.

Damasio, A. R. (2010). Y el cerebro creó al hombre. Ediciones Destino.

Danvila, I. \& Sastre, M. A. (2010). Inteligencia emocional: una revisión de la investigación. Cuadernos de Estudios Empresariales, 20, 107-126. https://bit. ly/3jVEWtj 
Domínguez, M. (2008). Factores determinantes en la gestión de recursos humanos en empresas de servicios que incorporan de manera sistemática nuevas tecnologías. Un estudio de caso en la comunidad valenciana. Pensamiento y Gestión, 24, 88-131. https:// bit.ly/2LKeBI1

Dulewicz, V. \& Higgs, M. (2004). Can emotional intelligence be developed? Human Resource Management, 15(1), 95111. https://bit.ly/2Z9300C

Duque, J.L., Garcia, M \& Hurtado, A. (2017). Influencia de la inteligencia emocional sobre las competencias laborales: un estudio empírico con empleados del nivel administrativo. Estudios Gerenciales, 33(144), 250-260. https://bit. ly/3d9nG1Z

Durán-Aponte, E. \& Durán-García, M. (2012). Competencias sociales y las prácticas profesionales. Vivencias y demandas para la formación universitaria actual. Cultura y Educación, 24 (1), 61-76. https://bit.ly/20xEyp8

Etchevers, N. (2008). La transmisión eficaz de comunicación emocional en la CMO, mediante un nuevo lenguaje complementado [Tesis de doctorado, Universidad de Salamanca]. Repositorio Universidad de Salamanca. https://gredos. usal.es/jspui/handle/10366/22641

Feldman, L. \& Blanco, G. (2006). Las emociones en el ambiente laboral: un nuevo reto para las organizaciones. Revista de la Facultad de Medicina, 29(2), 103-108. https://bit.ly/3tQ4EUq

Felman, R. (2006). Psicología (6. ${ }^{a}$ ed.). McGraw-Hill.

Fernandez, P. \& Ramos, N. (2002). Corazones inteligentes. Cairos.

George, J. M. (2000). Emotions and leadership: The role of emotional intelli- gence. Human Relations, 53(8), 1027 1055. https://bit.ly/379zMoa

Goleman, G. (1995). La inteligencia emocional. Editorial Javier Vergara.

Goleman, D. (1998). La práctica de la inteligencia emocional. Kairós.

Fernández-Aráoz, C. (2005). El desafío de contratar ejecutivos de alta dirección. En Inteligencia emocional en el trabajo. Cómo seleccionar, medir y mejorar la inteligencia emocional en individuos, grupos y organizaciones (pp. 185-196). Kairós.

García-Fernández, M. \& Giménez-Más, S. (2010). La inteligencia emocional y sus principales modelos: propuesta de un modelo integrador. Cuadernos del Profesorado, 3(6), 43-52.

Gazca, L. A., Zárate, X. M. \& Mota, A. A. (2020). Estudio de Motivación de Colaboradores en las Medianas Empresas en la Ciudad de Xalapa, México. Revista Gestión, Organizaciones y Negocios, 7(2), 1-11. https://doi. org/10.22579/23463910.207

Gracia, E. \& Martínez, I. M. (2004). El control de emociones en el trabajo: una revisión teórica del trabajo emocional. Jornades de Foment de la Investigació, 10, 1-18. http://hdl.handle. net/10234/78950

Gutiérrez, M. \& García, J. L. (2015). La comunicación emocional, una necesidad para una sociedad en crisis [ponencia]. I Congreso Internacional de Expresión y Comunicación Emocional. CIECE. Prevención de dificultades socio-educativas, Sevilla España. http://congreso.us.es/ciece/Virtual_02.pdf

Herrera, F., Ramírez, M. I., Roa, J. M. \& Herrera, I. (2004). Tratamiento de las creencias motivacionales en contextos educativos pluriculturales. Revista 
Iberoamericana de Educación, 34(1), 1-21. https://rieoei.org/RIE/article/ view/2885

llies, R. \& Judge, T. A. (2005). Goal Regulation Across Time: The Effects of Feedback and Affect. Journal of Applied Psychology, 90(3), 453-467. https://bit. Iy/3jN53T8

Jiménez, A. (2018). Inteligencia emocional. En Curso de Actualización Pediatría (pp. 457-469). Lúa Ediciones 3.0.

Jiménez-Ortega, L., Espuny, J., de Tejada, P. H., Vargas-Rivero, C. \& Martín-Loeches, M. (2017). Subliminal Emotional Words Impact Syntactic Processing: Evidence from Performance and Event-Related Brain Potentials. Frontiers in Human Neuroscience, 11(192). https://bit.ly/2LMonTV

Jordan, P. J., Ashkanasy, N. M., Härtel, C. E. J. \& Hooper, G. S. (2002). Workgroup Emotional Intelligence: Scale Development and Relationship to Team Process Effectiveness and Goal Foculs. Human Resource Management Review, 12(2), 195-214. https://bit.ly/3jL4vgq

Law, K. S., Wong, C. S., Huang, G. \& Li, X. (2008). The effects of emotional intelligence on job performance and life satisfaction for the research and development scientists in China. Asia Pacific Journal Management, 25, 51-69. https://bit.ly/3d9Gfn4

Labarca, F.A. \& Estaba, R. (2010). Inteligencia emocional como herramienta gerencial para la toma de decisiones de los directores del Comando General del Ejército Nacional Bolivariano. ClCAG: Revista del Centro de Investigación de Ciencias Administrativas y Gerenciales, 7(2), 63-80. https://bit.ly/3tSAwb4

Ljungholm, D. P. (2014). Emotional intelligence in organizational behaviour.
Economics, Management \& Financial Markets, 9(3), 128-133. https://bit. ly/3aklvHr

Lewis, K. M. (2000). When Leaders Display Emotion: How Follovers Respond to negative Emotional Expression of Male and Female Leaders. Journal of Organizational Behavior, 21(2), 221234. https://bit.ly/3pdyxuh

Locke, E. A. (2005). Why emotional intelligence is an invalid concept. Journal of Organizational Behavior, 26(4), 425431. https://doi.org/10.1002/job.318

Langhorn S. (2004). How emotional intelligence can improve management performance. International Journal of Contemporary Hospitality Management, 16(4), 220-230. https://bit.ly/3jL4yZE

Lopes, P. N., Côte, S. \& Salovey, P. (2006). An ability model of emotional intelligence: Implications for assessment and training. En Linking emotional intelligence and performance at work: Current research evidence with individuals and groups (pp. 53-80). Lawrence Erlbaum Associates Publishers.

López, E., Pulido, M. \& Augusto, J. M. (2013). Inteligencia emocional en el trabajo. Sintesis.

Loria, A. (2019). La toma de decisiones: El rol de las emociones. Centro de Neurociencias Sociales. https://bit.ly/3pdMHeP

Mayer, J. D., Caruso, D. \& Salovey, P. (1999). Emocional Intelligence: meets tradicional Standard for an intelligence. Intelligence, 27, 267-298.

Mayer, J. D. \& Salovey, P. (1990). The intelligence of emocional intelligence. Intelligence, 17, 43-44.

Mayer, J.D. \& Salovey, P. (1997). What is Emotional Intelligence? En Emotional 
Development and Emotional Intelligence (pp. 3-31). BasicBooks.

Martin, A. (2013). La inteligencia emocional: una herramienta necesaria para el liderazgo. Concepto y componentes. Revista de investigaciones UNAD, 12, 149-157.

Matthews, G., Zeidner, M. \& Roberts, R. (2002). Emotional intelligence: Science and myth? The MIT Press.

Mohammad, F. N., Chai, L. T., Aun, L. K. \& Migin, M. W. (2014). Emotional intelligence and turnover intention. International Journal of Academic Research, 6(4), 211-220. https://bit.ly/3aXkfsQ

O'Boyle, E. H., Jr., Humphrey, R. H., Pollack, J. M., Hawver, T. H. \& Story, P. A. (2011). The relation between emotional intelligence and job performance: A meta-analysis. Journal of Organizational Behavior, 32, 788-818. https://doi. org/10.1002/job.714

Pineda Henao, A. E. (2020). La comunicación organizacional en la gestión empresarial: retos y oportunidades en el escenario digital. Revista GEON (Gestión, Organizaciones Y Negocios), 7(1), 9-25. https://doi. org/10.22579/23463910.182

Peña, H. C. \& Villón, S. G. (2018). Motivación Laboral. Elemento Fundamental en el Éxito Organizacional. Instituto Internacional de Investigación y Desarrollo Tecnológico Educativo, 3(7), 177-192. https://bit.ly/3pdME2D

Pérez, A., Cruz, J. A., Guatemala, A. M. \& Juárez, V. (2018). Importancia de los pronósticos en la toma de decisiones en las MIPYMES. Revista Gestión, Organizaciones y Negocios, 5(1), 97-114. https://bit.ly/2OzaY2w

Ponti, F. \& Cabañas, B. (2004). Inteligencia emocional: la hora de la verdad. Lo que piensan los directivos españoles sobre la revolución en la inteligencia propuesta por Goleman. Capital Humano, 177, 78-80.

Prati, L., Douglas, C., Ferris, G., Ammeter, A. \& Buckley, M. (2003). Emotional intelligence, leadership effectiveness and team outcomes. The International Journal of Organizational Analysis, 11(1), 21-40. https://doi.org/10.1108/ eb028961

Ramirez, J. \& Zavaleta, V. (2018). Inteligencia Emocional y Desempeño Laboral en una Empresa Constructora Privada. 2017. Revista Ciencia y Tecnología, 14(4), 67-79. https://bit.ly/3tSualx

Rangarajan, R. \& Jayamala, C. (2014). Impact of Emotional Intelligence on Employee Performance-An Epigrammatic Survey. Sumedha Journal of Management, 3(1), 76-81.

Ryback, D. (1998). Trabaje con su inteligencia, los factores emocionales al servicio de la gestión empresarial y el liderazgo efectivo. Editorial EDAF, S.A.

Robbins, S. \& Judge, T. A. (2017). Comportamiento organizacional. Editorial Pearson.

Robbins, S. \& Judge, T.A. (2013). Introducción al comportamiento organizativo. Editorial Pearson Educación.

Robbins, S. \& Judge, T. A. (2010). Introducción al comportamiento organizativo. Editorial Pearson Educación.

Robbins, S. \& Judge, T. A. (2005). Comportamiento organizacional. Pearson.

Rooy, D. L. V. \& Viswesvaran, C. (2004). Emotional Intelligence: A meta-analytic investigation of predictive validity and nomological net. Journal of Vocational Behavior, 65, 71-95. https://bit. ly/2NOpCis 
Sassha, A. \$ Tumaev, T. (2007). Economía y recursos humanos: la selección de personal. Perspectivas, 20, 29-44.

Seco, J. J. (2006). La Inteligencia Emocional aplicada a la Administración de Personal. Tec Empresarial, (1), 20-24.

Solano, A. (2013). Inteligencia Emocional en el Trabajo: Implicaciones y el Rol de la Psicología Laboral. Revista Humanitas, 10(1), 201-214.

Sternberg, R., Castejón, J., Prieto, M., Hautamäki, J. \& Grigorenko, E. (2001). Confirmatory factor analysis of the Sternberg triarchic abilities test in three international samples: An empirical test of the triarchic theory. European Journal of Psychological Assessment, 17, 1-16. https://doi.org/10.1027/10155759.17.1.1

Suárez, A. \& Trespalacios, J. A. (2011). Competencias emocionales de los directivos de PYMES de servicios españolas y su influencia en el aprendizaje organizativo. Revista Europea de Dirección y Economía de la Empresa, 20(2), 87-100.

Subirán, G. (2010). El 90\% del éxito de un líder depende de su inteligencia emocional. Las competencias emocionales pueden desarrollarse. Gestión Práctica de Riesgos Laborales, 75, 12-15.

Thorndike, E. L. (1920). Intelligence and its use. Harper's Magazine, 140, 227 235

Toledo, F. \& Salvador, C. (2014). Manual de inteligencia emocional aplicada a la prevención de riesgos laborales. CROEM.
Torres-Flórez, D. (2020). La generación de valor entre las personas y las organizaciones. Revista Gestión, Organizaciones y Negocios, 7(1), 4-8. https://bit. ly/3qkqu6H

Trujillo, M. M. \& Rivas, L. A. (2005). Orígenes y evolución y modelos de inteligencia emocional. Innovar, 15(25), 9-24.

Tsai, W., Chen, C. \& Liu, H. (2007). Test of a Model Linking Employee Positive Moods and Task Performance. Journal of Applied Psychology, 92(6), 15701583. https://bit.ly/3rj0jAo

Voola, R., Carlson, J. \& West, A. (2004). Emotional intelligence and competitive advantage: examining the relationship from a resource-based view. Strategic Change, 13(2), 83-93. https:// doi.org/10.1002/jsc.667

Watkin, C. (2002). Developing emotional intelligence. International Journal of Selection and Assessment, 8(2), 89-92. https://bit.ly/3797s5b

Zárate, R \& Matviuk, S. (2010). La inteligencia emocional y el sector financiero colombiano. Revista EAN, (69), 148165. https://bit.ly/2ZpvTaB

Zardeni, Z. \& Yousefi, A. (2012). The role of emotional intelligence on workforce agility in the workplace. International Journal of Psychological Studies, 4(3), 48-61. https://doi.org/10.5539/ijps. v4n3p48 